\title{
РЕШЕНИЕ ИНТЕГРАЛЬНЫХ УРАВНЕНИЙ С ЭКСПОНЕНЦИАЛЬНЫМИ ЯДРАМИ
}

\section{1. Введение}

В данной работе дается алгоритм решения уравнения вида

$$
x(t)=\int_{0}^{H} \sum_{j=1}^{n} a_{j} \mathrm{e}^{-b_{j}|t-s|} x(s) d s+a_{0} \mathrm{e}^{b_{0} t},
$$

где

$$
\begin{gathered}
a_{j}, b_{j}>0, b_{0} \neq \pm b_{j}(j=1, \ldots, n), \\
\sum_{j=1}^{n} \frac{a_{j}}{b_{j}} \leqslant \frac{1}{2} .
\end{gathered}
$$

К уравнению (1) можно прийти, приближенно решая, например, одно из основных уравнений теории переноса излучения в однородной среде - уравнение Милна (см. [ [ $\left.\left.{ }^{1}\right]\right)$

$$
x(t)=\frac{\lambda}{2} \int_{0}^{H} E(|t-s|) x(s) d s+a_{0} \mathrm{e}^{b_{0} t} .
$$

Здесь $\lambda(0 \leqslant \lambda \leqslant 1)$ - вероятность выживания кванта излучения при столкновении с частицей среды,

$$
E(t)=\int_{1}^{\infty} \frac{\mathrm{e}^{-t s}}{s} d s=\int_{0}^{1} \frac{1}{\mu} \mathrm{e}^{-\frac{t}{\mu}} d \mu
$$

- интегральная экспонента. При изучении переноса излучения в неоднородной среде (в разорванной облачности) вместо (3) становится справедливым (см. $\left.\left[{ }^{2}\right]\right)$ уравнение вида

$$
x(t)=\int_{0}^{H}\left[l_{1} E\left(\lambda_{1}|t-s|\right)+l_{2} E\left(\lambda_{2}|t-s|\right)\right] x(s) d s+a_{0} \mathrm{e}^{b_{0} t} .
$$

Функция $E(t)$ в ходе решения уравнения (3) или (3’), как правило, сглаживается ввиду наличия у нее логарифмической особенности при $t=0$. Один из возможных путей заключается в замене интеграла в определении $E(t)$ какой-нибудь квадратурной формулой 


$$
\int_{0}^{1} f(\mu) d \mu \approx \sum_{j=1}^{n} \alpha_{j} f\left(\mu_{j}\right), 0<\mu_{1}<\mu_{2}<\ldots<\mu_{n} \leqslant 1, \alpha_{j}>0, \sum_{j=1}^{n} \alpha_{j}=1 .
$$

Таким образом,

$$
E(t) \approx \sum_{j=1}^{n} \frac{\alpha_{j}}{\mu_{j}} \mathrm{e}^{-\frac{t}{\mu j}}
$$

и, например, уравнение (3) заменяется уравнением (1), в котором

$$
a_{j}=\frac{\lambda}{2} \frac{\alpha_{j}}{\mu_{j}}, \quad b_{j}=\frac{1}{\mu_{j}} \quad(j=1, \ldots, n) .
$$

Заметим, что условие (2) здесь выполняется: из $\lambda \leqslant 1$ следует

$$
\sum_{j=1}^{n} \frac{a_{j}}{b_{j}}=\frac{\lambda}{2} \sum_{j=1}^{n} \alpha_{j}=\frac{\lambda}{2} \leqslant \frac{1}{2} .
$$

При этом случаю $\lambda=1$ (непоглощающая среда) соответствует равенство в (2). Поэтому критический случай

$$
\sum_{j=1}^{n} \frac{a_{j}}{b_{j}}=\frac{1}{2}
$$

представляет особый интерес.

Условие (2) гарантирует однозначную разрешимость уравнения (1) и равномерную сходимость метода последовательных приближений. Действительно, норма интегрального оператора $T$ уравнения (1) в пространстве $C[0, H]$ непрерывных на $[0, H]$ функций вычисляется по формуле (см. $\left.\left[{ }^{3}\right]\right)$

$$
\|T\|=\max _{0 \leqslant t \leqslant H} \int_{0}^{H} \sum_{j=1}^{n} a_{j} \mathrm{e}^{-b_{j}|t-s|} d s=\int_{0}^{H} \sum_{j=1}^{n} a_{j} \mathrm{e}^{-b_{j}\left|\frac{H}{2}-s\right|} d s,
$$

откуда

$$
\|T\|<2 \int_{0}^{\infty} \sum_{j=1}^{n} a_{j} \mathrm{e}^{-b y s^{\prime}} d s^{\prime}=2 \sum_{j=1}^{n} \frac{a_{j}}{b_{j}} \leqslant 1 .
$$

В пользу метода последовательных приближений для уравнения (1) говорит и то, что в принципе все интегрирования при начальном приближении $x_{0}(t)=a_{0} \mathrm{e}^{b_{0} t}$ удается провести точно без привлечения квадратурных формул. Однако точный счет громоздок, и при выполнении равенства (2') сходимость последовательных приближений очень медленная, особенно при больших $H$ (при $H \rightarrow \infty$ попадаем на спектр).

В $\left[{ }^{4}\right]$ разработан метод механических квадратур решения уравнения (1) - интеграл в уравнении (1), в свою очередь, заменяется какойнибудь квадратурной формулой.

Предлагаемый ниже метод решения уравнения (1) нам представляется более эффективным, особенно при небольших $n$.

\section{2. Решение уравнения (1)}

Посмотрим, как действует интегральный оператор $T$ уравнения (1) на экспоненциальные функции. При $d \neq b_{j},-b_{j}(\mathrm{j}=1, \ldots, n)$ несложный счет дает 


$$
T \mathrm{e}^{d t}=-\sum_{j=1}^{n} \frac{2 a_{j} b_{j}}{d^{2}-b_{j}^{2}} \mathrm{e}^{d t}-\sum_{j=1}^{n} \frac{a_{j}}{d+b_{j}} \mathrm{e}^{-b j t}+\sum_{j=1}^{n} \frac{a_{j} \mathrm{e}^{\left(d-b_{j}\right) H}}{d-b_{j}} \mathrm{e}^{b / t},
$$

т. е., применяя $T$ к функции $\mathrm{e}^{d t}$, получаем ту же функцию (с новым коэффициентом) и еще $2 n$ экспоненциальные функции вида $\mathrm{e}^{b j t}$ и $\mathrm{e}^{-b t t}$, которые оператор $T$ переводит в более сложные. Сказанное (а также некоторые другие соображения) наводит на мысль искать решение уравнения (1) в виде

$$
x(t)=c_{0} \mathrm{e}^{b_{0} t}+\sum_{k=1}^{2 n} c_{k} \mathrm{e}^{d_{k} t}
$$

с неизвестными пока $c_{k}$ и $d_{k}$, но такими, что $\left|d_{k}\right| \neq b_{j}(k=1, \ldots, 2 n$; $j=1, \ldots, n)$. Вопрос о существовании решения в указанной форме решается подстановкой (5) в уравнение (1) и приравниванием после применения формулы (4) коэффициентов при линейно независимых функция $\mathrm{e}^{b_{0} t}, \mathrm{e}^{ \pm b s t}, \mathrm{e}^{d k t}$. В результате приходим к следующим условиям на $c_{k}$ и $d_{k}$ :

$$
\begin{aligned}
& a_{0}-\sum_{j=1}^{n} \frac{2 a_{j} b_{j}}{b_{0}^{2}-b_{j}^{2}}=c_{0}, \\
& \sum_{k=1}^{2 n} \frac{c_{k}}{d_{k}+b_{j}}+\frac{c_{0}}{b_{0}+b_{j}}=0 \quad(j=1, \ldots, n), \\
& \sum_{k=1}^{2 n} \frac{c_{k} \mathrm{e}^{\left(d_{k}-b_{j}\right) H}}{d_{k}-b_{j}}+\frac{c_{0} \mathrm{e}^{\left(b_{0}-b_{j}\right) H}}{b_{0}-b_{j}}=0 \quad(j=1, \ldots, n), \\
& \sum_{j=1}^{n} \frac{2 a_{j} b_{j}}{d_{k}^{2}-b_{j}^{2}}=-1 \quad(k=1, \ldots, 2 n) .
\end{aligned}
$$

Из (6) однозначно определяется $c_{0}$. Если $d_{1}, \ldots, d_{2 n}$ уже найдены, и они попарно различны, $\left|d_{k}\right| \neq b_{j}$, то $c_{1}, \ldots, c_{2 n}$ легко находятся из линейной системы (7). Условия (8) говорят о том, что вместе с $d_{k}$ в решение (5) входит и $-d_{k}$, причем $x_{k}=d_{k}^{2}(k=1, \ldots, n)$ определяются из уравнения

$$
\sum_{j=1}^{n} \frac{2 a_{j} b_{j}}{x-b_{j}^{2}}+1=0
$$

Из условия (2) следует, что (8') имеет ровно $n$ попарно различных вещественных неотрицательных решений $x_{1}, \ldots, x_{n}$. Действительно, умножением на $\left(x-b_{1}^{2}\right) \ldots\left(x-b_{n}^{2}\right)$ левая часть уравнения преобразуется к многочлену степени $n$, а значит, существует не более $n$ решений. С другой стороны, из поведения функции $y=\sum_{j=1}^{n} \frac{2 a_{j} b_{j}}{x-b_{j}^{2}}$ ясно (см. рисунок), что уравнение (8') имеет решение в каждом из интервалов * $\left[\left(0, b_{1}^{2}\right),\left(b_{1}^{2}, b_{2}^{2}\right), \ldots,\left(b_{n-1}^{2}, b_{n}^{2}\right)\right]$. При этом, если в (2) выполняется строгое неравенство, все решения $x_{1}, \ldots, x_{n}$ положительны, если же имеет место равенство $\left(2^{\prime}\right)$, то одно решение нулевое, остальные положительные.

* Здесь мы считаем, что $b_{1}<b_{2}<\ldots<b_{n}$. 
Вид функции

$$
y=\sum_{j=1}^{n} \frac{2 a_{j} b_{j}}{x-b_{j}^{2}}
$$

(к решению уравнения $\left.\left(8^{\prime}\right)\right)$.

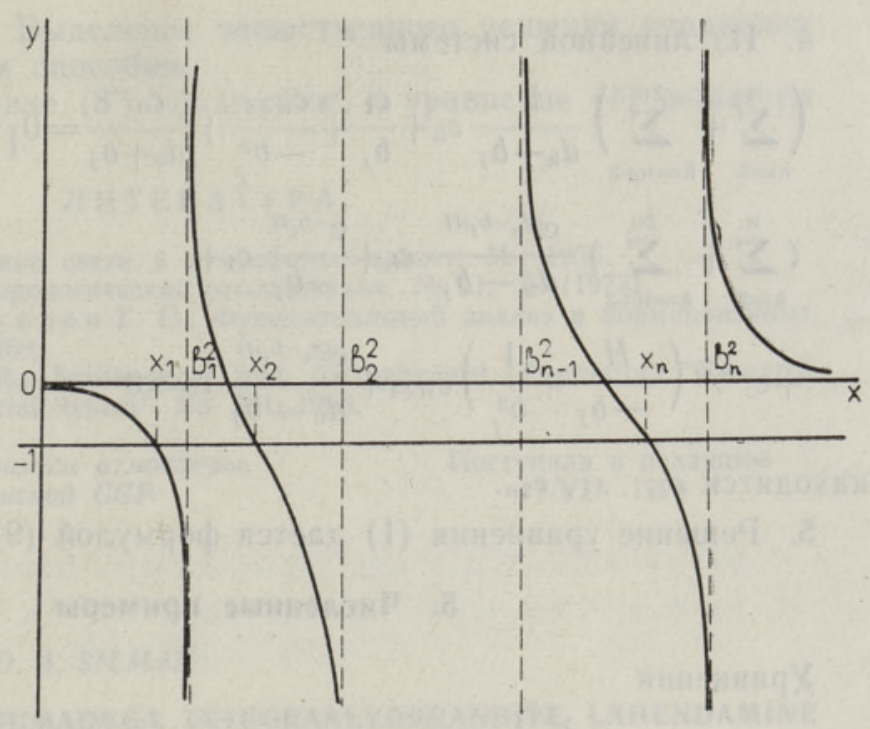

\section{3. Случай поглощающей среды (строгое неравенство в (2))}

В этом случае на основе изложенного получаем следующий алгоритм решения уравнения (1).

1. Составляется уравнение (8'). Методом Ньютона (или каким-нибудь другим итерационным методом) находятся его положительные решения $x_{1}, \ldots, x_{n}$. За начальные приближения принимаются соответственно $\frac{1}{2} b_{1}^{2}, \frac{1}{2}\left(b_{1}^{2}+b_{2}^{2}\right), \ldots, \frac{1}{2}\left(b_{n-1}^{2}+b_{n}^{2}\right)$ или числа, получаемые решением квадратных уравнений, составленных из (8') с сохранением соответствующих двух членов.

2. Вычисляются $d_{k}=\sqrt{x_{k}}, d_{k+n}=-\sqrt{x_{k}}(k=1, \ldots, n)$.

3. Из (6) определяется $c_{0}$.

4. Из линейной системы (7) находятся $c_{1}, \ldots, c_{2 n}$.

5. Решение уравнения (1) дается формулой (5).

\section{4. Случай непоглощающей среды (равенство (2'))}

В этом случае, как уже отмечалось, первый из корней $x_{k}$ $(k=1, \ldots, n)$ равен нулю, вместе с ним $d_{1}=d_{n+1}=0$, определитель системы (7) равен нулю, и алгоритм п. 3 не срабатывает.

Решение уравнения (1) следует разыскивать в виде

$$
x(t)=c_{0} \mathrm{e}^{b_{0} t}+c_{1}+c_{n+1} t+\left(\sum_{j=2}^{n}+\sum_{j=n+2}^{2 n}\right) c_{k} \mathrm{e}^{d_{k} t} .
$$

Повторяя выкладки п. 2, приходим к следующему алгоритму.

1. Составляется уравнение (8'). Методом Ньютона (или каким-нибудь другим итерационным методом) находятся его положительные решения $x_{2}, \ldots, x_{n}$. За начальные приближения выбираются соответственно числа $\frac{1}{2}\left(b_{1}^{2}+-b_{2}^{2}\right) \ldots, \frac{1}{2}\left(b_{n-1}^{2}+b_{n}^{2}\right)$.

2. Вычисляются $d_{1}=d_{n+1}=0, d_{k}=\sqrt{x_{k}}, d_{k+n}=-\sqrt{x_{k}}(k=2, \ldots, n)$.

3. Из (6) определяется $c_{0}$. 
4. Из линейной системы

$$
\begin{aligned}
& \left(\sum_{k=2}^{n}+\sum_{k=n+2}^{2 n}\right) \frac{1}{d_{k}+b_{j}} c_{k}+\frac{c_{1}}{b_{j}}+\frac{c_{n+1}}{-b_{j}^{2}}+\frac{c_{0}}{d_{0}+b_{j}}=0 \quad(j=1, \ldots, n), \\
& \left(\sum_{k=2}^{n}+\sum_{k=n+2}^{2 n}\right) \frac{\mathrm{e}^{\left(d_{k}-b_{j}\right) H}}{d_{k}-b_{j}} c_{k}+\frac{\mathrm{e}^{-b_{j} H}}{-b_{j}} c_{1}+ \\
& -\mathrm{e}^{-b_{j} H}\left(\frac{H}{-b_{j}}+\frac{1}{b_{j}^{2}}\right) c_{n+1}+\frac{\mathrm{e}^{\left(d_{0}-b_{j}\right) H}}{d_{0}-b_{j}} c_{0}=0
\end{aligned}
$$

находятся $c_{1}, \ldots, c_{2 n}$.

5. Решение уравнения (1) дается формулой (9).

\section{5. Численные примеры}

Уравнения

$$
x(t)=\frac{\lambda}{2} \int_{0}^{1} E(|t-s|) x(s) d s+a_{0} \mathrm{e}^{b_{0} t}, \quad a_{0}=\frac{1}{4}, \quad b_{0}=3
$$

H

$$
\begin{gathered}
x(t)=\frac{3}{2} \int_{0}^{1} E(3|t-s|) x(s) d s+a_{0} \mathrm{e}^{b_{0} t}, \\
a_{0}=\mathrm{e}^{-3,4642}, \quad b_{0}=3,4642
\end{gathered}
$$

заменялись уравнениями вида (1) указанным в п. 1 способом, при этом использовалась формула средних прямоугольников для разных $n$. Уравнение (10) решалось по алгоритму п. 3 при различных $\lambda<1$, а уравнение (11), соответствующее критическому случаю, - по алгоритму п. 4. Некоторые результаты расчетов, выполненных на ЭВМ «Минск-32», приведены в табл. 1 и 2.

Таблица 1

Таблица 2

Решение уравнения (10) при $\lambda=0,4$

Решение уравнения (11)

\begin{tabular}{l|ll}
\hline \multirow{2}{*}{$t$} & \multicolumn{2}{|c}{$x(t)$} \\
\cline { 2 - 3 } & $n=5$ & $n=10$ \\
\hline 0 & 0,9391 & 0,9388 \\
0,1 & 1,2229 & 1,2280 \\
0,2 & 1,5176 & 1,5194 \\
0,3 & 1,8482 & 1,8482 \\
0,4 & 2,2341 & 2,2328 \\
0,5 & 2,6937 & 2,6907 \\
0,6 & 3,2464 & 3,2413 \\
0,7 & 3,9124 & 3,9065 \\
0,6 & 4,7026 & 4,7058 \\
0,9 & 5,6008 & 5,6336
\end{tabular}

\begin{tabular}{l|ll}
\hline \multirow{2}{*}{$t$} & \multicolumn{2}{|c}{$x(t)$} \\
\cline { 2 - 3 } & $n=4$ & $n=6$ \\
\hline 0 & 0,5219 & 0,5208 \\
0,1 & 0,8674 & 0,8665 \\
0,2 & 1,1552 & 1,1523 \\
0,3 & 1,4268 & 1,4232 \\
0,4 & 1,6870 & 1,6828 \\
0,5 & 1,9335 & 1,9290 \\
0,6 & 2,1598 & 2,1552 \\
0,7 & 2,3534 & 2,3487 \\
0,8 & 2,4902 & 2,4849 \\
0,9 & 2,5087 & 2,5084
\end{tabular}

\section{6. Замечания}

1. Алгоритм решения уравнения (1) несложно получить и тогда, когда (2) нарушается. В таком случае уравнение (8') имеет $n-1$ положительных решений и одно отрицательное $x_{1}$, которому соответствуют 
два числа $d_{k}= \pm i \sqrt{\left|\overline{x_{1}}\right|}$. Выделение вещественного решения уравнения (1) происходит обычным способом.

2. При $n=1$ уравнение (8') квадратное, и уравнение (1) решается точно, что известно из [ $\left.{ }^{1}\right]$.

\section{ЛИ Т ЕРА Т У Р А}

1. С оболев В. В., Рассеяние света в атмосферах планет, М., 1956.

2. В а й н и к о Г. М., Метеорологические исследования, № 21, 38 (1973).

3. К а н торов ич Л. В., А к ил о в Г. П., Функциональный анализ в нормированных пространствах, М., 1959.

4. Arett E. H., L o e ser R., Smithsonian Inst. Astrophysical Observatory "Research in Space Science. Special Report", No. 201, 1966.
Ннститут астрофизики и физики атмосферы Академии наук Эстонской ССР
Поступила в редакцию 7/VII 1975

G. VAINIKKO, L. KARPENKO, A. SILMAN

\section{EKSPONENTSIAALSETE TUUMADEGA INTEGRAALVORRANDITE LAHENDAMINE}

Esitatakse võrrandi (1) lahendusalgoritm. Lahendit otsitakse kujul (5), suurused $c_{k}$ ja $d_{k}$ määratakse süsteemist (7) ja võrrandist (8). On käsitletud neelava ja mitteneelava keskkonna juhtu.

\section{G. VAINIKKO, L. KARPENKO, A. SHILMAN}

\section{SOLUTION OF INTEGRAL EQUATIONS WITH EXPONENTIAL KERNELS}

In the present paper we give an algorithm for solving the equation (1). The solution is presented in the form (5) where the unknown quantities $c_{k}, d_{k}$ are found from the system (7) and the equation (8). We have considered the algorithms in the case of absorbing as well as nonabsorbing media. 